\section{ScienceDirect}

Materials Today: Proceedings 11 (2019) 695-699 materialstoday:
PROCEDDINGS

www.materialstoday.com/proceedings

$2^{\text {nd }}$ International Conference on Emerging Materials: Characterization and Application

(EMCA-2017)

\title{
Characterization of Tempering Behaviour of Modified 9Cr-1Mo Steel by Ultrasonic Lamb wave Mixing
} Avijit Kr. Metya ${ }^{\mathrm{a}, *}$, Soumitra Tarafder ${ }^{\mathrm{b}}$, Krishnaan Balasubamaniam ${ }^{\mathrm{c}}$

${ }^{a}$ AMP Division,CSIR- National Metallurgical Laboratory, Jamshedpur-831007,India,

${ }^{b}$ MTE Division, CSIR-National Metallurgical Laboratory, Jamshedpur-831007, India

${ }^{c}$ CNDE, Mechanical Engineering Department, IIT Madras, Chennai- 600036, India

\begin{abstract}
This work demonstrates the use of mixing of ultrasonic Lamb waves to characterize the tempering behaviour in metallic plate. Lamb wave mixing has been used to measure the generated second harmonic during tempering of mod.9Cr-1Mo steel plate. As-received material is normalized at $1080^{\circ} \mathrm{C}$ and then tempered in the temperature range of $600^{-} 850^{\circ} \mathrm{C}$ with a step size of $50^{\circ} \mathrm{C}$ for $1.5 \mathrm{hrs}$ and followed by furnace cooling. Lamb wave mixing technique has been used to assess the tempering behaviour of this material. Nonlinear ultrasonic parameter $\beta$ which is the ratio of $2^{\text {nd }}$ harmonic amplitude to the multiplication of the fundamental amplitudes is determined from the mixing wave at each temperature and correlated with microstructural characteristics. It is seen that this nonlinear acoustic parameter $(\beta)$ is sensitive towards coherency strain generated between precipitate and matrix during tempering.
\end{abstract}

(C2019 Elsevier Ltd. All rights reserved.

Selection and peer-review under responsibility of Conference Committee of the $2^{\text {nd }}$ International Conference on Emerging Materials: Characterization and Application (EMCA-2017).

Keywords: Lamb wave mixing, Nonlinearity parameter, Tempering, Mod. 9Cr-1Mo steel

* Corresponding author. Tel.: +91-9199993308

E-mail address: avijit@nmlindia.org; avijit353@gmail.com

2214-7853@2019 Elsevier Ltd. All rights reserved.

Selection and peer-review under responsibility of Conference Committee of the $2^{\text {nd }}$ International Conference on Emerging Materials:

Characterization and Application (EMCA 2017). 
1. Introduction

Nonlinear ultrasonic (NLU) technique has already shown to be a promising technique to characterize the microstructural characteristics during degradation/ heat-treatment [1-3]. Conventional ultrasonic technique generally relies on the linear behavior of stress-strain relationship; whereas, NLU derives itself from the nonlinear Hooke's law that is dominated by higher order elastic constants. Initial pure sinusoidal wave gets distorted and generates higher order harmonics when encounters nonlinearities within the materials. But, the major problem in NLU technique is to distinguish between the material nonlinearity and other external nonlinearities from instrumentations, couplant etc. as the main objective is to evaluate the nonlinearity generated within material during deformation/ heat-treatment. Works have been started on collinear or non-collinear mixing of bulk waves within materials under certain resonance conditions to generate a third wave which carries the information of the nonlinearity from the mixing zone $[4,5]$. In this work, two Lamb wave modes of different frequencies $\left(\omega_{1} \& \omega_{2}\right)$ are allowed to mix under certain resonance conditions within the material to generate third type of harmonic waves of frequencies $\left(\omega_{1} \pm \omega_{2}\right)$. The generated mixing wave carries the information of the material nonlinearities from the mixing zone. So, attempt has been made to characterize the tempering behavior of modified $9 \mathrm{Cr}-1 \mathrm{Mo}$ steel by mixing two Lamb waves and the result has been compared to our earlier work on study of tempering behavior of this same material using nonlinear Lamb wave technique [6]. Initial feasibility study of Lamb wave mixing has been performed by D J Lee et al. [7]. Some aspects of guided wave mixing have also been discussed by $\mathrm{C} \mathrm{J}$ Lissenden et al. [8].

\section{Experiment}

The mixing of multiple waves in the presence of nonlinearities within a material generates combinational frequencies. Two mutually interacting waves of frequencies $\mathrm{fl}$ and $\mathrm{f} 2$ will generate other combinational frequencies of $2 \mathrm{fl}, 2 \mathrm{f} 2,(\mathrm{fl} \pm \mathrm{f} 2)$ and higher harmonics accordingly. Single mode waves are desirable for mixing due to multimode and dispersive nature of Lamb waves. In this work, S0 mode was chosen of frequencies $0.73 \mathrm{MHz}$ and $0.41 \mathrm{MHz}$ due to their non-dispersive nature to detect $(0.73+0.41)=1.14 \mathrm{MHz}$. The as-received plate was machined to make rectangular shape of thickness $2 \mathrm{~mm}$. The other dimensions were kept same as in [6]. Higher harmonic measurement by mixing of two Lamb wave modes was done using high power pulser RAM 5000 from RITEC Inc. Fig. 1 shows the schematic of the experimental set-up for the higher harmonic measurement using Lamb wave mixing. A centre frequency of $1 \mathrm{MHz}$ broadband transducer was used as transmitter and $2.25 \mathrm{MHz}$ broadband transducer was used as receiver.

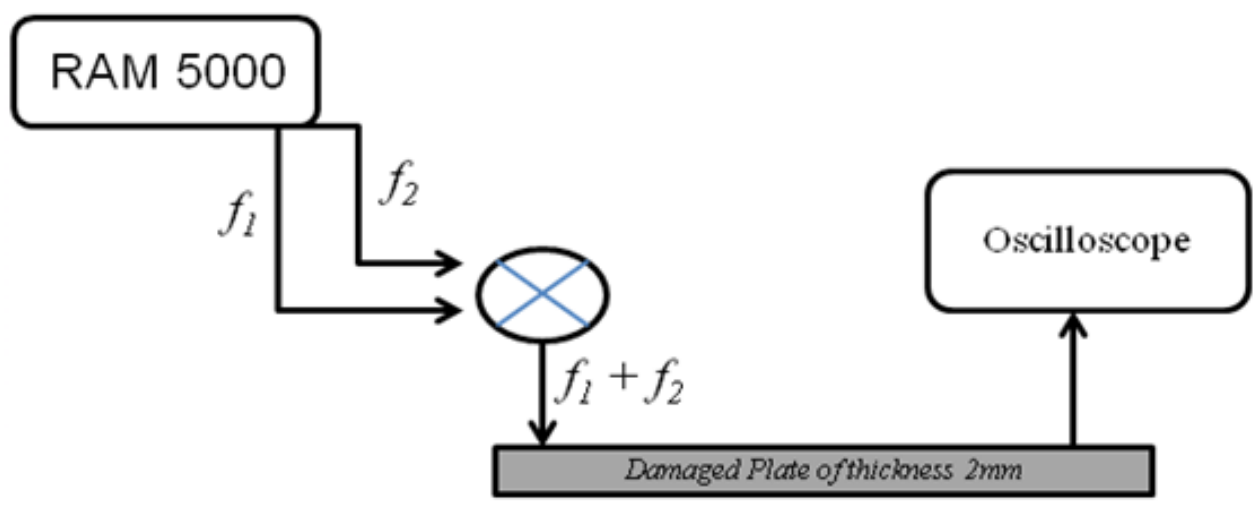

Fig. 1: Schematic of the experimental set-up for Lamb wave mixing technique

\section{Results and Discussion}

Figure-2 shows a typical multimode, non-stationary type received mixing signal and the Fast Fourier Transformation of the received signal shows the presence of sum frequency component $(1.14 \mathrm{MHz})$ along with two fundamental frequencies $(0.41 \& 0.73 \mathrm{MHz})$ [fig.-3]. 


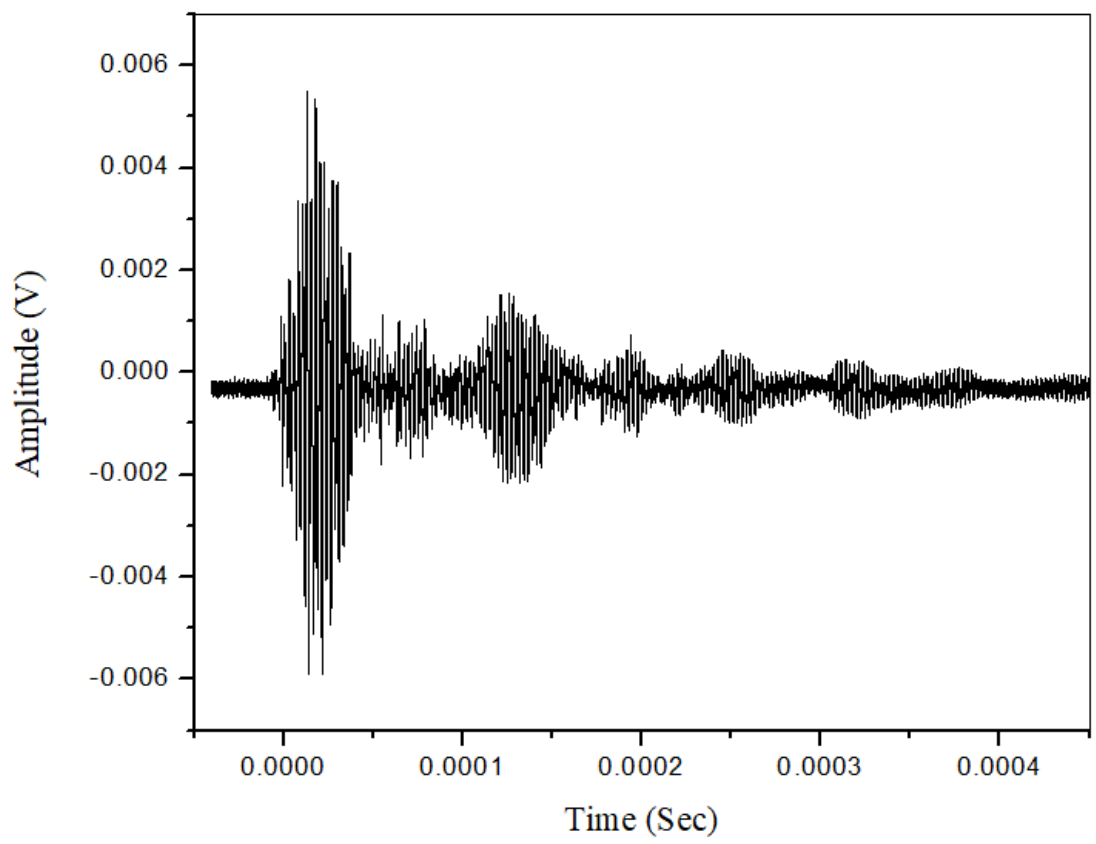

Fig. 2: Typical multi-mode non-stationary type received signal
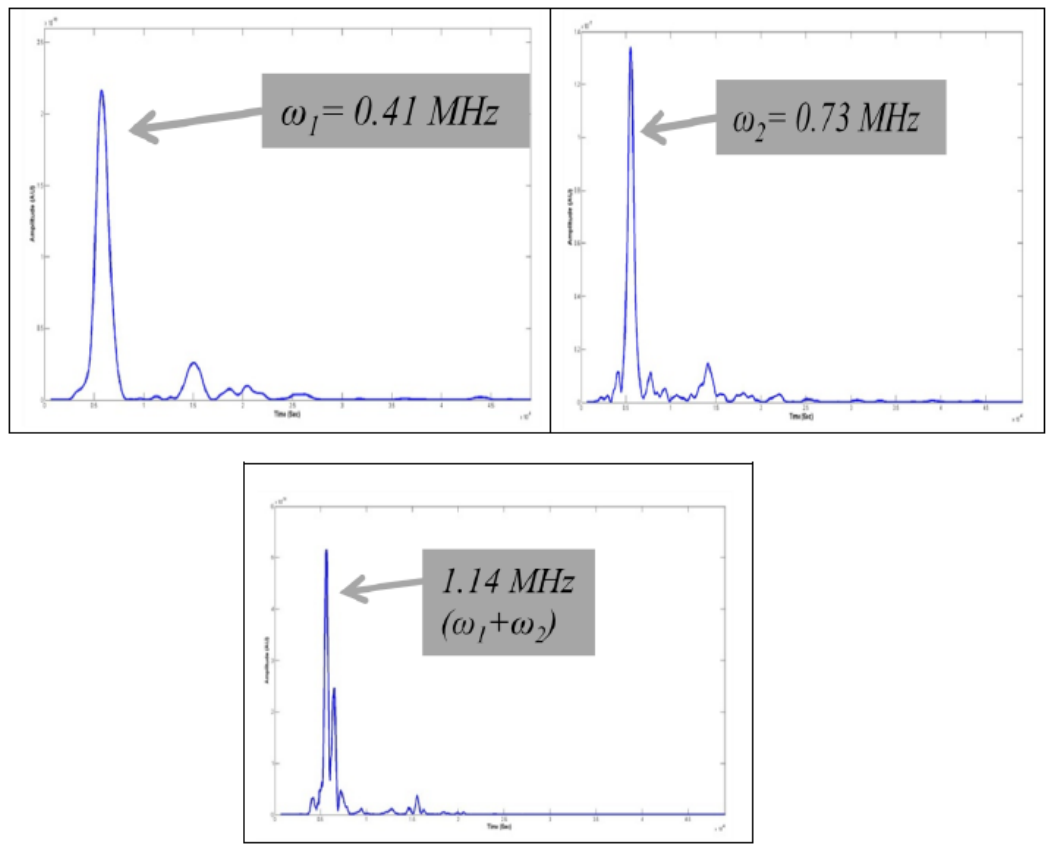

Fig. 3: Fast Fourier Transformation of the received signal shows the presence of sum frequency component

Evolution of various types of carbides (mainly $\mathrm{M}_{23} \mathrm{C}_{6}[\mathrm{M}=\mathrm{Cr}]$ and $\mathrm{MX}[\mathrm{M}=\mathrm{V} . \mathrm{Nb}$ and $\mathrm{X}=\mathrm{C}, \mathrm{N}]$ type) may be seen to occur during tempering [6]. Precipitation of these types of carbides controls the coherency strain between 
precipitates and matrix which in turn makes changes in the nonlinear acoustic parameter. Fig.- 4 shows the variation of relative change in acoustic nonlinearity parameter using Lamb wave mixing and hardness with tempering temperatures. Relative change in $\beta$ has been defined as $\left(\frac{\theta-\theta_{n}}{\theta_{n}}\right)$, where $\beta=\left(\frac{A_{k}}{\beta_{1} \beta_{2}}\right)$ and $\beta_{0}$ is the nonlinear ultrasonic parameter at initial condition (as-received); $\mathrm{A}_{1}, \mathrm{~A}_{2}$ and $\mathrm{A}_{3}$ are the amplitude of the two fundamental frequencies and sum frequency components respectively [9].

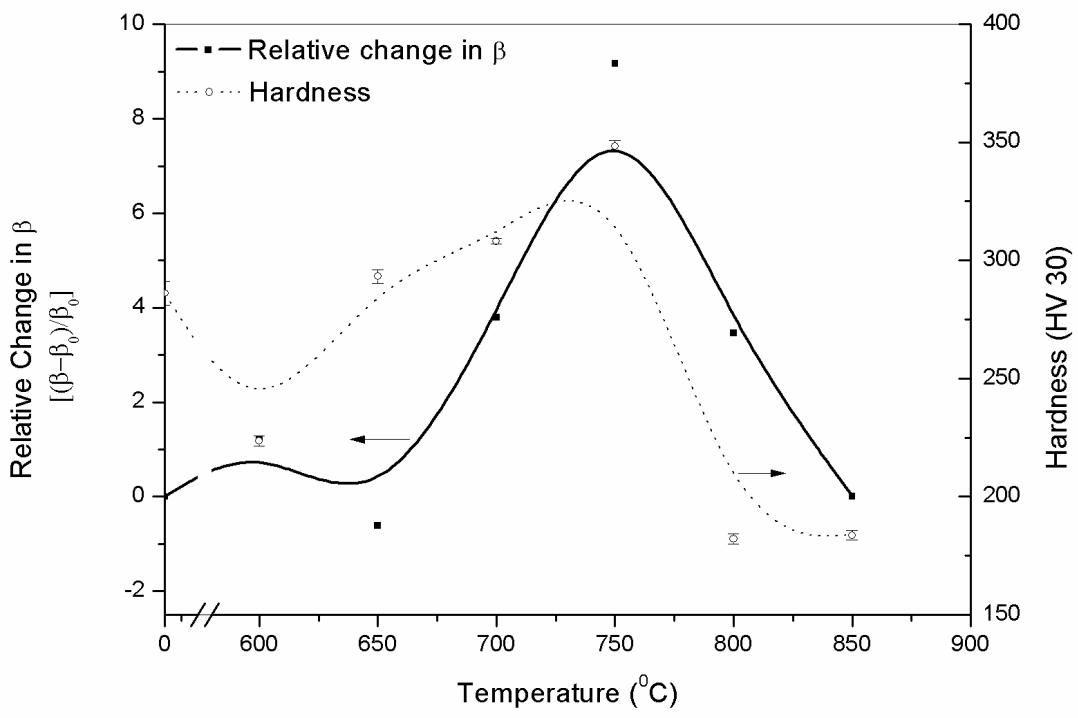

Fig. 4: Variation of relative change of acoustic nonlinearity parameter evaluated from Lamb wave mixing along with hardness with tempering temperatures

Figure- 4 shows that variation of relative change in $\beta$ is much smoother than the variation that was obtained in our earlier work [6]. Here also, it is seen that precipitate matrix coherency strain controls the variation in $\beta$ and $750^{\circ} \mathrm{C}$ could be the optimum tempering temperature for obtaining maximum strength/ flow stress of this material [6].

\section{Conclusion}

Effect of tempering temperatures on the Lamb wave mixing signal was studied. Two Lamb wave modes were allowed to mix under resonant conditions to generate a third type of wave of sum frequency of the fundamental waves.

The variation of relative change in $\beta$ is smoother than the variation of $\beta$ obtained from direct nonlinear Lamb wave measurement.

\section{Acknowldgements}

The authors would like to thank Director, CSIR- NML for his kind permission to use infrastructural facilities to carry out this work and to present this work in $2^{\text {nd }}$ International Conference on Emerging Materials: Characterization and Application (EMCA-2017).

\section{References}

[1] D.C. Hurkey, D. Balzar, P.T. Purtscher, K.W. Hollman, "Nonlinear ultrasonic parameter in quenched martensitic steel", J. Appl. Phys. 83 (9) (1998) 4584-4588.

[2] W.L. Morris, O. Buck, R.V. Inman, Acoustic harmonic generation due to fatigue in high strength aluminium, J. Appl. Phys. 50 (11) (1979) 6737-6741. 
[3] J.H. Cantrell, W.T. Yost, Determination of precipitate nucleation and growth rates from ultrasonic harmonic generation, Appl. Phys. Lett. 77 (13) (2000) 1952-1954.

[4] G L Jones, "Interaction of elastic waves in an isotropic solid", J. Acoust. Soc. Am, 35(1) (1963), 5-10.

[5] A J Croxford, P D Wilcox, B W Drinkwater, "The use of non-collinear mixing for nonlinear ultrasonic detection of plasticity and fatigue", J. Acoust. Soc. Am., 126(5) (2009), 117-122.

[6] Avijit Kr. Metya, Mainak Ghosh, N. parida, and Krishnan Balasubramaniam, "Effect of tempering temperartures on nonlinear Lamb wave signal of modified 9Cr-1Mo steel”, Materials Characterization, 107(2015)14-22.

[7] D J Lee, Y Cho, W Li, “A feasibility study for Lamb wave mixing nonlinear technique”, AIP Conference Proceedings, (2014), 662-666.

[8] C J Lissenden, Y Liu, V K Chillara, G Choi, and H Cho, "Nonlinear guided wave mixing for localized material state characterization", Physics Procedia, 70 (2015) 668-671.

[9] J Jingpin, M Xiangji, H Cunfu, and W Bin, "Nonlinear lamb wave mixing technique for micro- crack detection in plates", NDT \& E Int., 85 (2017) 63-71 